\title{
Kelvin probe force microscopy work function characterization of transition metal oxide crystals under ongoing reduction and oxidation
}

\author{
Dominik Wrana ${ }^{* 1}$, Karol Cieślik ${ }^{1}$, Wojciech Belza ${ }^{1}$, Christian Rodenbücher ${ }^{2}$, \\ Krzysztof Szot ${ }^{3,4}$ and Franciszek Krok ${ }^{1}$
}

\author{
Full Research Paper \\ Address: \\ ${ }^{1}$ Marian Smoluchowski Institute of Physics, Jagiellonian University, \\ Lojasiewicza 11, 30-348 Krakow, Poland, ${ }^{2}$ Institute of Energy and \\ Climate Research (IEK-3), Forschungszentrum Jülich GmbH, 52425 \\ Jülich, Germany, ${ }^{3}$ Peter Grünberg Institute (PGI-7), \\ Forschungszentrum Jülich $\mathrm{GmbH}, 52425$ Jülich, Germany and \\ ${ }^{4}$ August Chelkowski Institute of Physics, University of Silesia, 40-007 \\ Katowice, Poland \\ Email: \\ Dominik Wrana* - dominik.wrana@uj.edu.pl \\ ${ }^{*}$ Corresponding author \\ Keywords: \\ Kelvin probe force microscopy (KPFM); reduction and oxidation; \\ $\mathrm{SrTiO}_{3}$; $\mathrm{TiO}$ nanowires; $\mathrm{TiO} / \mathrm{SrTiO}_{3}$ heterostructure; transition metal \\ oxides; work function \\ Beilstein J. Nanotechnol. 2019, 10, 1596-1607. \\ doi:10.3762/bjnano. 10.155 \\ Received: 19 April 2019 \\ Accepted: 09 July 2019 \\ Published: 02 August 2019 \\ This article is part of the thematic issue "Advanced atomic force \\ microscopy II". \\ Guest Editor: T. Glatzel \\ (C) 2019 Wrana et al.; licensee Beilstein-Institut. \\ License and terms: see end of document.
}

\begin{abstract}
Controlling the work function of transition metal oxides is of key importance with regard to future energy production and storage. As the majority of applications involve the use of heterostructures, the most suitable characterization technique is Kelvin probe force microscopy (KPFM), which provides excellent energetic and lateral resolution. In this paper, we demonstrate precise characterization of the work function using the example of artificially formed crystalline titanium monoxide (TiO) nanowires on strontium titanate $\left(\mathrm{SrTiO}_{3}\right)$ surfaces, providing a sharp atomic interface. The measured value of 3.31(21) eV is the first experimental work function evidence for a cubic TiO phase, where significant variations among the different crystallographic facets were also observed. Despite the remarkable height of the TiO nanowires, KPFM was implemented to achieve a high lateral resolution of $15 \mathrm{~nm}$, which is close to the topographical limit. In this study, we also show the unique possibility of obtaining work function and conductivity maps on the same area by combining noncontact and contact modes of atomic force microscopy (AFM). As most of the real applications require ambient operating conditions, we have additionally checked the impact of air venting on the work function of the $\mathrm{TiO} / \mathrm{SrTiO}_{3}(100)$ heterostructure, proving that surface reoxidation occurs and results in a work function increase of $0.9 \mathrm{eV}$ and $0.6 \mathrm{eV}$ for $\mathrm{SrTiO}_{3}$ and $\mathrm{TiO}$, respectively. Additionally, the influence of adsorbed surface species was estimated to contribute $0.4 \mathrm{eV}$ and $0.2 \mathrm{eV}$ to the work function of both structures. The presented method employing KPFM and local conductivity AFM for the characterization of the work function of transition metal oxides may help in understanding the impact of reduction and oxidation on electronic properties, which is of high importance in the development of effective sensing and catalytic devices.
\end{abstract}




\section{Introduction}

Transition metal oxides are viewed today as some of the most promising materials in various fields, ranging from (photo)catalysis [1], hydrogen production [2], resistive switching [3] and organic electronics [4,5] to so-called thermoelectric power generators [6]. The performance of all of the abovementioned applications is extremely sensitive to the work function (WF) of the active oxide layer. As a vast majority of applications are nowadays based on oxide heterostructures, not only is macroscopic information of the work function needed (which may be provided by averaging techniques such as ultraviolet photoelectron spectroscopy (UPS)) but also spatial resolution on the nanoscale. Driven by its remarkable lateral and energetic resolution, Kelvin probe force microscopy (KPFM, also known as scanning Kelvin probe microscopy, SKPM) is the tool of choice for the precise measurement of the WF across oxide heterostructures, which is a technique that has not been fully exploited to date. In recent years, KPFM has proved to be superior for many cases in both fundamental research and applications, such as the identification of adsorption geometries of molecules on oxide surfaces [7], probing energetics of electron transfer within single molecules [8] and operation of prototypical electronic devices, such as perovskite solar cells [9] or $\mathrm{Ti} / \mathrm{TiO}_{x} / \mathrm{Ti}$ memristive devices [10]. Of the two KPFM operation modes, frequency modulation (FM) has proven to be more suitable for the investigation of oxide nanostructures (due to the higher lateral resolution) as compared to amplitude modulation (AM) [11] Therefore, in our study, we present the advantages and limitations of the FM-KPFM technique using the example of a newly discovered $\mathrm{TiO} / \mathrm{SrTiO}_{3}(100)$ (metal/insulator) heterostructure, which has potentially high technological relevance [12].

Now it would be justified to introduce both $\mathrm{TiO}$ and $\mathrm{SrTiO}_{3}$ oxides, highlighting the differences and similarities between those two structures. Based on the electronic conduction, most transition metal oxides could be classified as insulators or semiconductors. However, due to the plethora of available valence states in which a cation can be, many transition metal oxides may also exhibit metallic conductivity. Here, a huge advantage over other materials is the possibility of oxides that self-dope via the introduction of oxygen vacancies [13], which is also a reason why there are not many reliable experimental studies on the work function of transition metal oxides (with one notable exception [14]). Strontium titanate, $\mathrm{SrTiO}_{3}$, is a perfect example of a semiconductor with a wide bandgap of $3.2 \mathrm{eV}$ and also a model perovskite oxide. $\mathrm{Ti}^{4+}$ cations provide no electrons for the d-band, which can participate in conductivity. Strontium titanate finds many applications as a dielectric ceramic material [15] but also in various heterostructures, with exotic electronic states, e.g., a two-dimensional electron gas (2DEG) on the interface with $\mathrm{LaAlO}_{3}[16,17]$. On the other side of the transition metal oxides spectrum lies titanium monoxide, $\mathrm{TiO}$, in which the titanium atoms adopt the valence state $2+$, contributing to the formation of $\mathrm{d}^{2}$ electrons. Hence, $\mathrm{TiO}$ is a d-band conductor with a room temperature conductivity of $3500 \Omega^{-1} \mathrm{~cm}^{-1}$, which slowly decreases with temperature [18]. There is a growing interest in such metallic oxides, not only as oxide electrodes but also in medicine, since reduced $\mathrm{TiO}$ nanostructures exhibit a strong absorbance of light over a broad spectrum, which is of potential use in novel tumor therapy [19]. In addition, in the previous year, a profound interest was focused on the superconductivity of various $\mathrm{TiO}$ structures [20,21]. According to those, rock-salt $\mathrm{TiO}$ is a type-II superconductor with a superconductivity transition temperature $\left(T_{\mathrm{c}}\right)$ of $5.5 \mathrm{~K}$, which is higher than previously reported results [22]. As a result of its electronic structure, titanium monoxide nanoparticles find further application in heterogeneous catalysis, e.g., for the hydrogenation of styrene [1].

Here we show the properties of a bulk-like crystalline rock-salt $\mathrm{TiO}$ phase, unlike previous studies on the crystallography and electronic structure of $\mathrm{TiO}$, which were based on defective thin films formed on various surfaces, e.g., TiC(100) [23] or $\mathrm{TiO}_{2}(110)$ [24]. $\gamma$-TiO is the high-temperature phase with a NaCl-type (rock-salt-type, B1) cubic structure $(F m-3 m$, $a=4.184 \AA$ ), displaying a wide range of nonstoichiometric values [25]. Our study contains first measurements of electrical conductivity and the work function of crystalline $\mathrm{TiO}$ and its response to the ambient air reoxidation. This is all compared to the work function of another relevant oxide, $\mathrm{SrTiO}_{3}(100)$, showing similarities and differences.

The present manuscript is organized as follows: after the introduction of our $\mathrm{TiO} / \mathrm{SrTiO}_{3}(100)$ system, a combined conductivity and work function study from the same surface area is presented, showing the possibility of obtaining full information on the electronic properties when the KPFM technique is accompanied by local conductivity atomic force microscopy (LC-AFM). This is followed by a discussion of the significant variations of the WF within cubic TiO nanowires, the estimation of the KPFM resolution and the differences between $\mathrm{TiO}_{2}$ and $\mathrm{SrO}$ terminations of $\mathrm{SrTiO}_{3}(100)$. The last part of the study is dedicated to the discussion of the work function response of both $\mathrm{TiO}$ and $\mathrm{SrTiO}_{3}$ surfaces upon oxidation via ambient air exposure, in order to provide insight into the effect of oxygen, water, and carbon dioxide interaction and therefore mimic the operation conditions in real life applications.

\section{Results and Discussion}

The thermal reduction of a $\mathrm{SrTiO}_{3}(100)$ crystal under reduced oxygen partial pressure (UHV conditions + an oxygen getter), 
assured by the extremely low oxygen partial pressure (ELOP) process, results in the formation of a network of ordered $\mathrm{TiO}$ nanowires on the (100) surface as shown in Figure 1. Heavily reducing conditions enable not only the removal of oxygen from the crystal but additionally trigger an incongruent sublimation of strontium, resulting in the titanium enrichment of the surface [26]. This general process has also been observed for the broader class of transition metal oxides, such as $\mathrm{CaTiO}_{3}$ or $\mathrm{BaTiO}_{3}$. The titanium monoxide surface layer crystallizes in the form of nanowires oriented along the main crystallographic directions of $\mathrm{SrTiO}_{3}(100)$, with a length of up to $10 \mu \mathrm{m}$, a width of a few hundred nanometers and a height of tens of nanometers - see Figure 1 . Their size could be easily tuned either by the temperature or time of reduction [12]. The perfect crystallographic order of $\mathrm{TiO}$ nanostructures is confirmed by the transmission electron microscopy (TEM) measurements in the highangle annular dark-field imaging scanning transmission electron microscope (HAADF-STEM) mode as we have recently shown in [12]. A closer look at $\mathrm{TiO}$ reveals the $\gamma$-TiO structure in a Fm-3m space group and $2.1 \AA$ unit cell, which stands in perfect agreement with subject literature values [27]. The high level of crystallinity of these nanowires is the result of the formation mechanism, which comprises incongruent strontium effusion from $\mathrm{SrTiO}_{3}$ and then a $\mathrm{TiO}$ structure growth via crystallographic shearing and diffusion (more details can be found in [12]). The abrupt $\mathrm{TiO} / \mathrm{SrTiO}_{3}$ interface and the $\mathrm{Ti}^{2+}$ to $\mathrm{Ti}^{4+}$ transition thereof make such a transition metal oxide heterostructure a promising candidate for various electronic properties and charge transfer investigations.

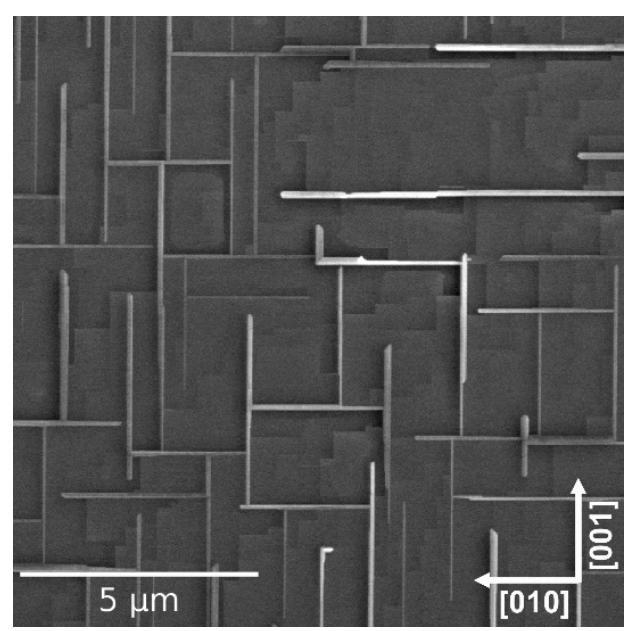

Figure 1: SEM images of an oriented network of titanium monoxide (TiO) nanowires on $\mathrm{SrTiO}_{3}(100)$. In between the terrace structure of $\mathrm{SrTiO}_{3}(100)$ can be seen.

Despite the similarities (both structures have a cubic crystallographic phase), there are profound differences in the electronic structure between the band insulator $\mathrm{SrTiO}_{3}$ and metallic TiO. Here there are two $3 \mathrm{~d}$ electrons per one $\mathrm{Ti}^{2+}$ divalent titanium ion, partially filling the metallic d-band in the energy diagram. From the orbital perspective, high conductivity is a consequence of the d-orbital overlap from the neighboring Ti sites. In the case of cubic TiO, the Ti-Ti distance is slightly above $2 \AA$, which is enough to have a significant overlap given the d-orbital extension. On the other hand, in the ideal $\mathrm{SrTiO}_{3}$ perovskite there are no d-electrons on $\mathrm{Ti}$ sites. Thus a $\mathrm{TiO}$ network on $\mathrm{SrTiO}_{3}$ constitutes a metallic nanowire array embedded in an insulator matrix, and to properly disentangle the electronic properties of both structures a technique with nanoscale resolution is needed. Indeed with the use of the LC-AFM technique, the conductivity of the developed nanostructures at the nanoscale can be characterized, providing the possibility of obtaining current maps as well as $I-V$ characteristics at a given spot. Figure 2a and Figure 2b show the topography and current maps of the $\mathrm{TiO}$ nanowire network on $\mathrm{SrTiO}_{3}(100)$. TiO has a higher conductivity than the surrounding $\mathrm{SrTiO}_{3}$ (STO) surface (the no current areas at nanowire edges are due to technical artefacts, such as wear of the coating of the conductive probe). To better illustrate the differences, $I-V$ characteristics of $\mathrm{TiO}$ and STO were collected and are presented in Figure 2c. Given the ohmic behavior at the $\mathrm{TiO}$ nanowire, the conductance of the whole system (tip + contact $+\mathrm{TiO}$ nanowire + interface $+\mathrm{STO}$ bulk + bottom electrode) can be estimated to $G_{\mathrm{LCAFM}}=10 \mu \mathrm{S}$. In contrast, the STO surface exhibits a one order of magnitude lower current of a rather semiconducting nature. It is noteworthy that the $\mathrm{STO}(100)$ surface had been thermally reduced up to $1150{ }^{\circ} \mathrm{C}$ in UHV beforehand, resulting in the formation of a high concentration of oxygen vacancies. Therefore, the conductivity is much higher than that of a pristine single crystal, which has been estimated via a comparable LC-AFM study to be around $10^{-16} \mathrm{~S}$ [28]. Here the observed changes of conductivity on the surface correlate directly with the work function differences as provided by the KPFM measurements taken at the very same area. This was possible by forcing the same conductive contact AFM tip to oscillate at higher harmonics to enter the FM-KPFM mode (more details can be found in [29]). Figure 2d,e presents the topography and work function of the same area. Differences in the WF are as high as $900 \mathrm{meV}$ between $\mathrm{TiO}$ and $\mathrm{SrTiO}_{3}$, in favor of $\mathrm{TiO}$. However, there is also a certain variation within the $\mathrm{TiO}$ and $\mathrm{SrTiO}_{3}$ structures, which will be discussed later. The bias sweep measurements presented in Figure $2 \mathrm{f}$ show reproducible Kelvin parabola with negative curvature for both structures. As up and down bias sweeps appear to follow the same curve, and there is no sign of charging or charge transfer. A comparative study of LC-AFM and $\mathrm{KPFM}$ of the $\mathrm{TiO} / \mathrm{SrTiO}_{3}$ structure enables a clear distinction between two materials of comparable conductivity but notably different work functions. The reason behind this is that 

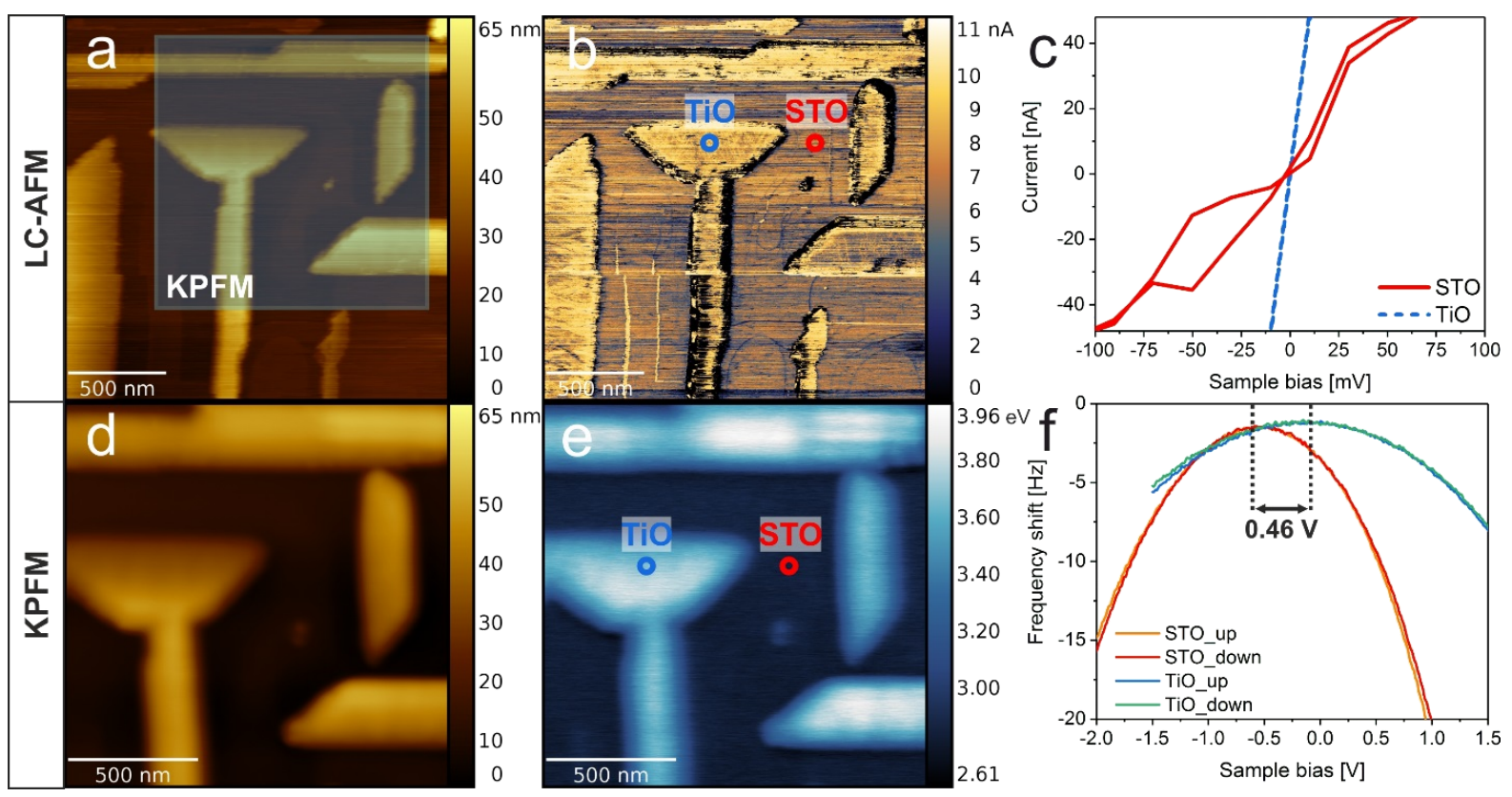

Figure 2: Electrical properties of $\mathrm{TiO}_{\mathrm{SrTiO}}(100)$ heterostructures. a), b) LC-AFM topography and current (PtIr-coated PPP-ContPt tip, $+1 \mathrm{mV}$ sample bias), c) $I-V$ characteristic (up and down sweeps) of marked areas on a TiO nanowire and $\mathrm{SrTiO}_{3}$ (STO) surface, d,e) KPFM topography and work function measurement of the very same area, and f) Kelvin parabola at the same TiO and STO structures.

although undoped $\mathrm{SrTiO}_{3}(100)$ is a band insulator, it could be easily self-doped with oxygen vacancies upon thermal reduction $[28,30]$. Reduction preferentially occurs at the surface, resulting in the reconstruction transformation from $(1 \times 1)$ to $(\sqrt{5} \times \sqrt{5}) \mathrm{R} 26.6^{\circ}$, and in the vicinity of extended defects in a crystal (dislocations) which act as easy conduction paths for electrons. Oxygen vacancy formation, and therefore $\mathrm{Ti}^{3+}$ valence, results in the appearance of new $t_{2 g}$ electron states within $\mathrm{Ti} 3 \mathrm{~d}$, which are below the conduction band of $\mathrm{SrTiO}_{3}(100)$ [31]. Consequently, a decrease of the WF is expected, as it was previously reported for $900{ }^{\circ} \mathrm{C}$ thermal annealing under UHV, where the WF of $\mathrm{SrTiO}_{3}(100)$ yielded 3.478(64) eV [30]. Hence, here a high conductivity of the reduced $\mathrm{SrTiO}_{3}(100)$ is measured and a work function of $3.12(18) \mathrm{eV}$, which is almost $1 \mathrm{eV}$ lower than previous $\mathrm{X}$-ray photoemission electron microscopy (XPEEM) and UPS studies (4.13 and $4.2 \mathrm{eV}$ ) for untreated oxide [32,33].

As stated before, KPFM investigations reveal certain variations in the work function value of $\mathrm{TiO}$ nanostructures. To illustrate this properly a 3D topography overprinted image with color scale representing the WF is shown in Figure 3a. The WF of
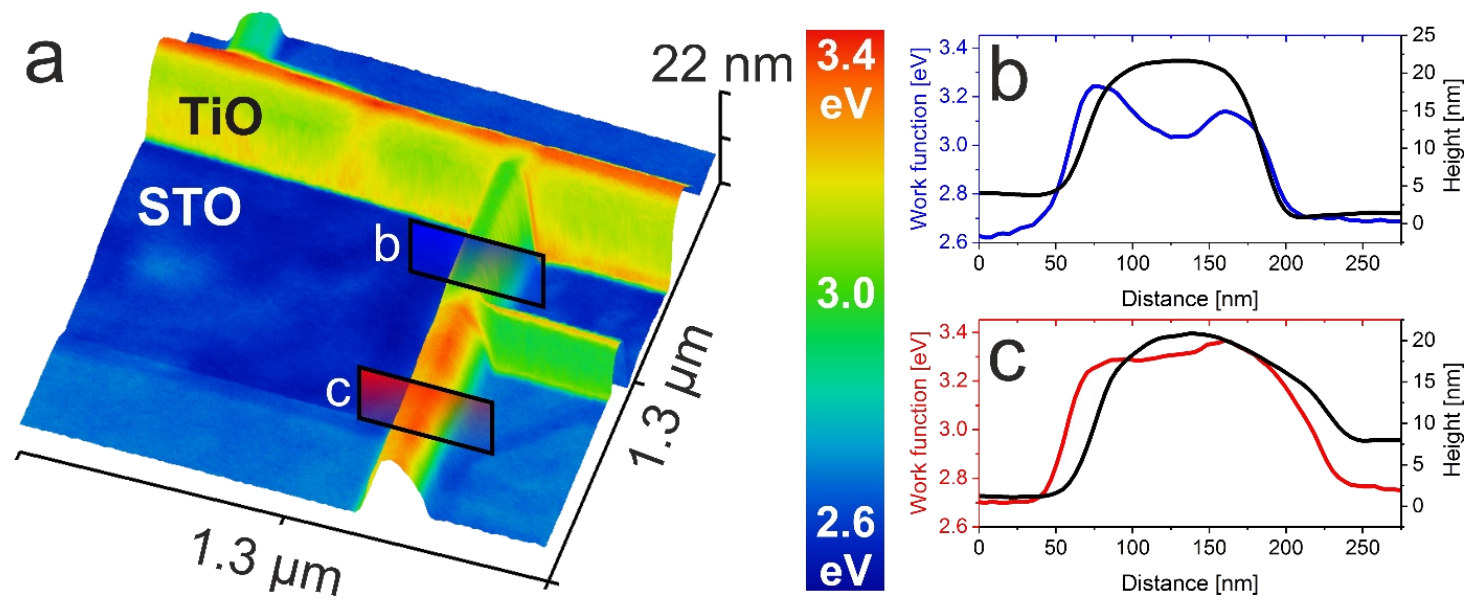

Figure 3: TiO facets influence the work function (WF) on the nanoscale. a) 3D view of the combined topography and the WF of $\mathrm{TiO}^{\mathrm{S}} \mathrm{SrTiO}$. b,c) $\mathrm{To}^{-}$ pography and work function profiles of two areas on the same TiO nanowire. 
titanium monoxide varies to as high as $300 \mathrm{meV}$, even within one nanowire. This is not an imaging artifact but rather a morphological-related feature, which is proved by the two profiles obtained for the same nanowire and shown in Figure 3b,c. The edges of the nanowires have a different work function from the top surface. This could be a consequence of the different facets of the cubic TiO crystal being exposed. Such an effect of facets having different WFs has to date been observed for many structures - the differences could be as high as $255 \mathrm{meV}$ measured in the case of $(\overline{1} \overline{1} \overline{2})$ and (110) surfaces of $\mathrm{CuGaSe}_{2}$ [34]. For the case of transition metal oxide crystals, XPEEM studies have proved that the WF of the $\mathrm{SrTiO}_{3}(111)$ face is higher than that of the (100) face by no less than $210 \mathrm{meV}$ [32]. Smaller differences in the range of $70 \mathrm{meV}$ were reported in the case of titanium dioxide (110) and (100) faces [35]. In the present case, the differences could be higher because the whole sample with $\mathrm{TiO}$ nanostructures was annealed up to $1150{ }^{\circ} \mathrm{C}$ and thus a possible reduction-driven non-stoichiometry occurs. From a geometrical perspective, the low index faces of cubic TiO, like (100), (110) or (210), have an equal number of protruding oxygen and titanium atoms, in contrast to the (111) face, where a whole plane is formed by either titanium or oxygen. To our knowledge, there are no subject literature studies on the stability or work function on such faces, but it is justified to assume certain differences may be present between those facets, which are also influenced by the preferential removal of oxygen during thermal reduction. As TiO nanowires have a height of tens of nanometers and tip convolution may play a role, a precise evaluation of the steep facets of a higher WF, as seen in Figure 3, is difficult. Based on the relative slope of a TiO surface it seems that the top surface typically adopts a (100) plane, though this requires further investigation.

An overlay of the work function on the topography map shown in Figure 3a provides additional information on the high lateral contrast of KPFM. The WF and morphology match almost completely, although height variations are of tens of nanometers. To investigate the resolution limits of KPFM imaging of oxide heterostructures with a complex topography, a set of images of parallel $\mathrm{TiO}$ nanowires was investigated, and the results are presented in Figure 4. As a measure of the potential resolution, we have used the ratio between the measured con-

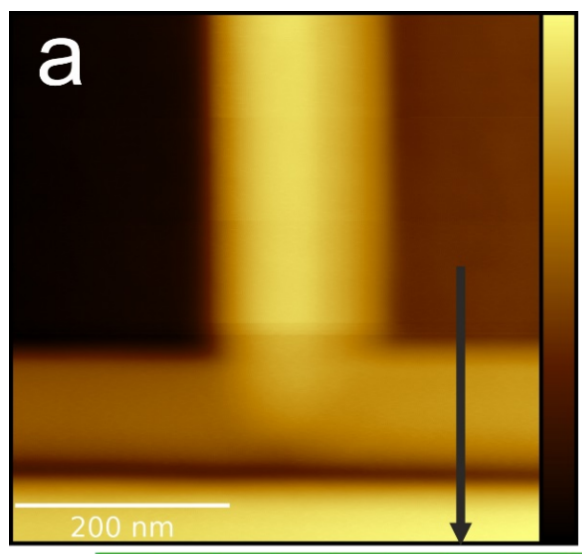

$20.0 \mathrm{~nm}$
18.0
16.0
14.0
12.0
10.0
8.0
6.0
4.0
0.0
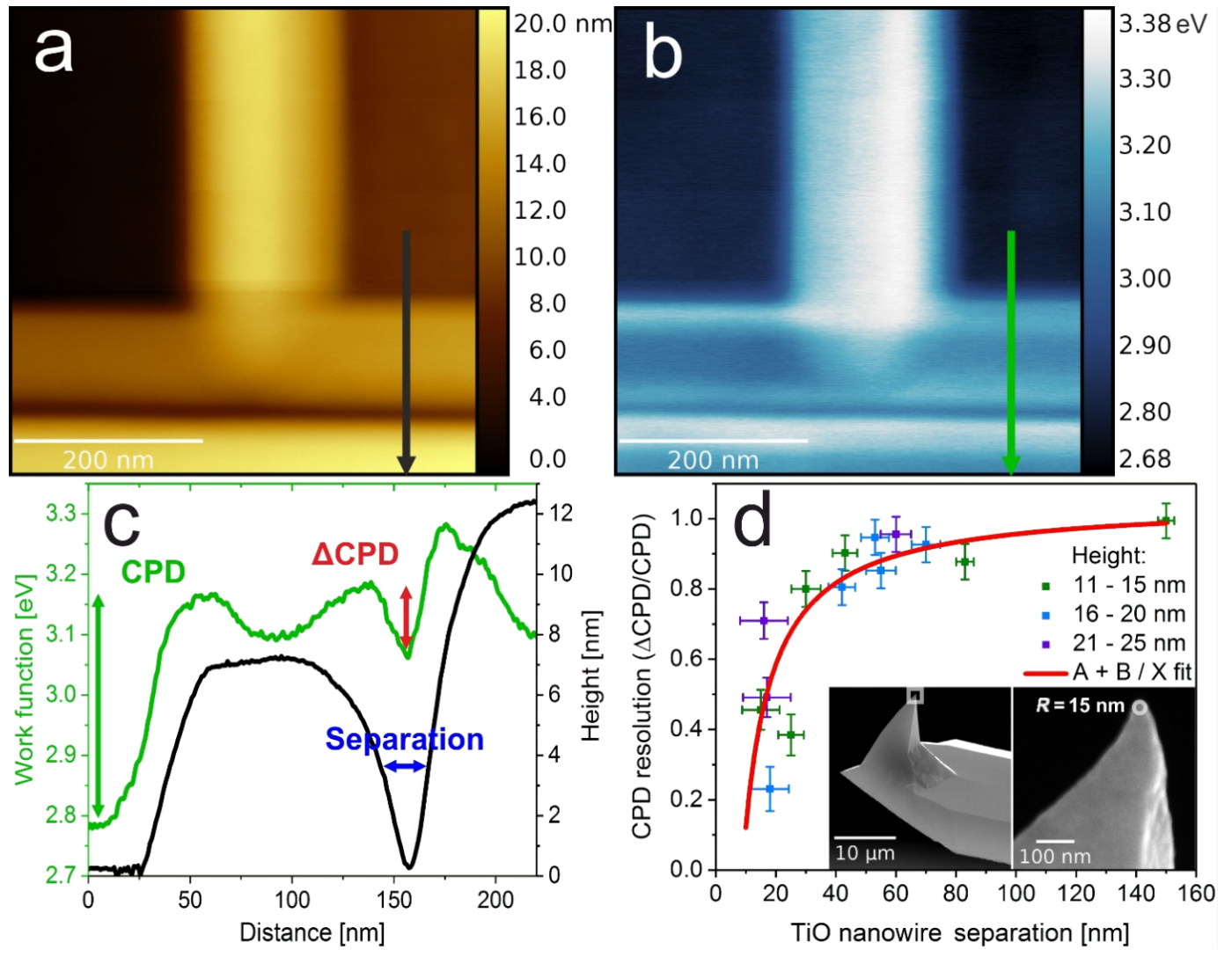

Figure 4: KPFM lateral resolution on high TiO/STO structures. a) Topography and b) work function of $\mathrm{TiO}$ nanowire array on $\mathrm{SrTiO}_{3}(100)$. c) $\mathrm{Height}$ (black line) and work function (green line) profiles of two adjacent TiO nanowires, showing high KPFM contrast. d) Dependence of the CPD resolution (estimated as $\triangle \mathrm{CPD} / \mathrm{CPD}$, see $\mathrm{c}$ ) on the separation between TiO nanowires, with $\mathrm{A}+\mathrm{B} / \mathrm{X}$ asymptote fit. Insets show the SEM images of the actual PtSi cantilever used in the experiments with a tip radius of $15 \mathrm{~nm}$. 
tact potential difference (CPD) decrease in between the TiO nanowires and the full CPD of TiO with respect to STO. For an ideal case, when the CPD reaches the value of STO in the gap between parallel nanowires, this measure adopts 1 , and 0 if there is no drop in CPD. The dependence of this value on the $\mathrm{TiO}$ nanowire separation (plotted in Figure 4d) provides information on the resolution limit. It follows an asymptotic behavior, with the correct CPD values measured when TiO nanowires are separated by more than $40 \mathrm{~nm}$. This stands in perfect agreement with the real tip radius of $15 \mathrm{~nm}$, which was measured for the same cantilever in high-resolution SEM (see Figure $4 d$ insets). Here, the use of uniformly PtIr-coated tips enables topography correlated artifacts to be avoided, unlike with some previous studies [36]. Taking into account the opening angle of about $40^{\circ}$ and the average nanowire height of $18(8) \mathrm{nm}$, the tip diameter at the level of the top surface of the nanowire would be about $40 \mathrm{~nm}$, meaning that the CPD resolution approaches the topography resolution, which is the ultimate physical limit of the FM-KPFM technique [11]. Similar results were obtained for the resolution estimation for $\mathrm{KBr}$ nanoislands of monoatomic thickness, where a resolution of 0.5 was obtained for $20 \mathrm{~nm}$ separation, as is the case in the following study [37]. The CPD resolution at the $\mathrm{TiO} / \mathrm{SrTiO}_{3}$ transition is insensitive to the nanowire height, following the same asymptotic behavior (see Figure 4d), unlike the situation for C60 islands on HOPG, where a spread of $50 \mathrm{~nm}$ was reported, while the topography resolution was $10 \mathrm{~nm}$ [38]. From our results, we can set the limit of the smallest separation of oxide nanostructures at $15 \mathrm{~nm}$ to obtain any CPD difference. As for the CPD resolution, the estimated value would be below $5 \mathrm{meV}$, due to the high mechanical stability and good conductivity of both platinum silicide and PtIr-coated tips.

Apart from the work function difference between the $\mathrm{TiO}$ and STO materials, we also found that the CPD/WF mapping of the STO(100) itself exhibits a nonuniform nature. This could be associated with two different exposed surfaces, as $\mathrm{SrTiO}_{3}(100)$ perovskite structure has two stable nonpolar terminations, $\mathrm{SrO}$ and $\mathrm{TiO}_{2}$, which both are present on the pristine surface, although $\mathrm{TiO}_{2}$ is more stable [39]. When annealed under reducing conditions, the $\mathrm{TiO}_{2}$ termination is promoted. Upon heavy reduction, there is further oxygen depletion which results in the formation of the $(\sqrt{5} \times \sqrt{5}) \mathrm{R} 26.6^{\circ}$ reconstruction, which we recently proved to be a Ti-enriched layer on $\mathrm{TiO}_{2}$-terminated $\mathrm{SrTiO}_{3}(100)[30,40]$. The electronic structures of both terminations are much different - the DFT calculated work function values are $1.92 \mathrm{eV}$ and $4.48 \mathrm{eV}$ for $\mathrm{SrO}$ and $\mathrm{TiO}_{2}$, respectively [14], obtained for the pure surfaces without vacancies.

In the present case of thermally reduced $\mathrm{SrTiO}_{3}(100)$, the dominant reconstruction is $(\sqrt{5} \times \sqrt{5}) \mathrm{R} 26.6^{\circ}$, which forms on the $\mathrm{TiO}_{2}$ termination, as proved by the scanning tunneling microscopy (STM) and low-energy electron diffraction (LEED) investigations (see Figure $5 \mathrm{~g}, \mathrm{~h}$ ). The surface is composed of two equiprobable orientations of $(\sqrt{ } 5 \times \sqrt{5}) \mathrm{R} 26.6^{\circ}$ domains, both rotated with respect to the $(1 \times 1)$ surface by 26.6 degrees. Those two domains are labeled A and B, both in LEED and STM images. Bright protrusions seen in the STM picture, which also decorate domain boundaries, are either oxygen vacancies/ vacancy clusters or Sr adatoms, according to the model [30]. The KPFM map reveals a non-homogeneous landscape of the work function - higher values are measured on terraces, whereas areas near the edges a reduced work function is observed (see Figure $5 b$ ). This could be directly represented by the histogram shown in Figure 5c. A clear difference of $\approx 23 \mathrm{meV}$ could be measured between the two areas. This effect is not purely topographical, since the WF differences are similar in the cases of a single step and multistep (12 atomic layers), as shown in Figure $5 \mathrm{~d}-\mathrm{f}$. Three possible mechanisms are possible to cause such difference: termination-, reconstruction- or reductionrelated. The first would be the termination variation within one terrace, with the higher WF values related to the $\mathrm{TiO}_{2}$ termination, which is dominant, and the lower to the SrO-enriched terrace edges, as previous KPFM studies showed the difference between air-annealed $\mathrm{SrTiO}_{3}$ terminations of $\approx 10 \mathrm{meV}$ [41]. However, for the case of heavily reduced $\mathrm{SrTiO}_{3}(100)$ the only observed termination is $\mathrm{TiO}_{2}$, given the step uniform heights of $\approx 4 \AA$. The second possible origin of the observed variation is the presence of multiple surface reconstructions, notwithstanding that the recorded LEED patterns and STM maps do not support such claims, showing clear $(\sqrt{5} \times \sqrt{5}) \mathrm{R} 26.6^{\circ}$ surfaces, even near the step edges (see Figure $5 \mathrm{~h}$ ). Finally, this difference is not likely to be caused by the electronic layer at step edges since it stretches away from the edges up to $100 \mathrm{~nm}$, which is too far to be attributed to local dipoles [42]. Simultaneously the oxygen removal is likely to be initiated at step edges, as it was as postulated by Martinez et al. [43] for a similar $\mathrm{TiO}_{2}$ (110) oxide. Hence for the severe reduction of $\mathrm{SrTiO}_{3}(100)$ a gradient of the average cation valence across one terrace may be expected, which can contribute to the observed small decrease in the WF near the step edges. In terms of the absolute work function of $\mathrm{TiO}_{2}$-terminated $\mathrm{SrTiO}_{3}(100)$, the obtained value of $3.12(18) \mathrm{eV}$, averaged across a number of images, is close to this postulated by DFT $(3.39 \mathrm{eV})$ for $\mathrm{TiO}_{2}$ termination with oxygen vacancies in the surface layer [14]. The difference is most likely caused by the higher density of oxygen vacancies, due to the heavy thermal reduction.

\section{Ambient air reoxidation of $\mathrm{TiO}$ and $\mathrm{SrTiO}_{3}$}

As the vast majority of applications of transition metal oxides is related to the oxygen activity and redox reactions on surfaces, 


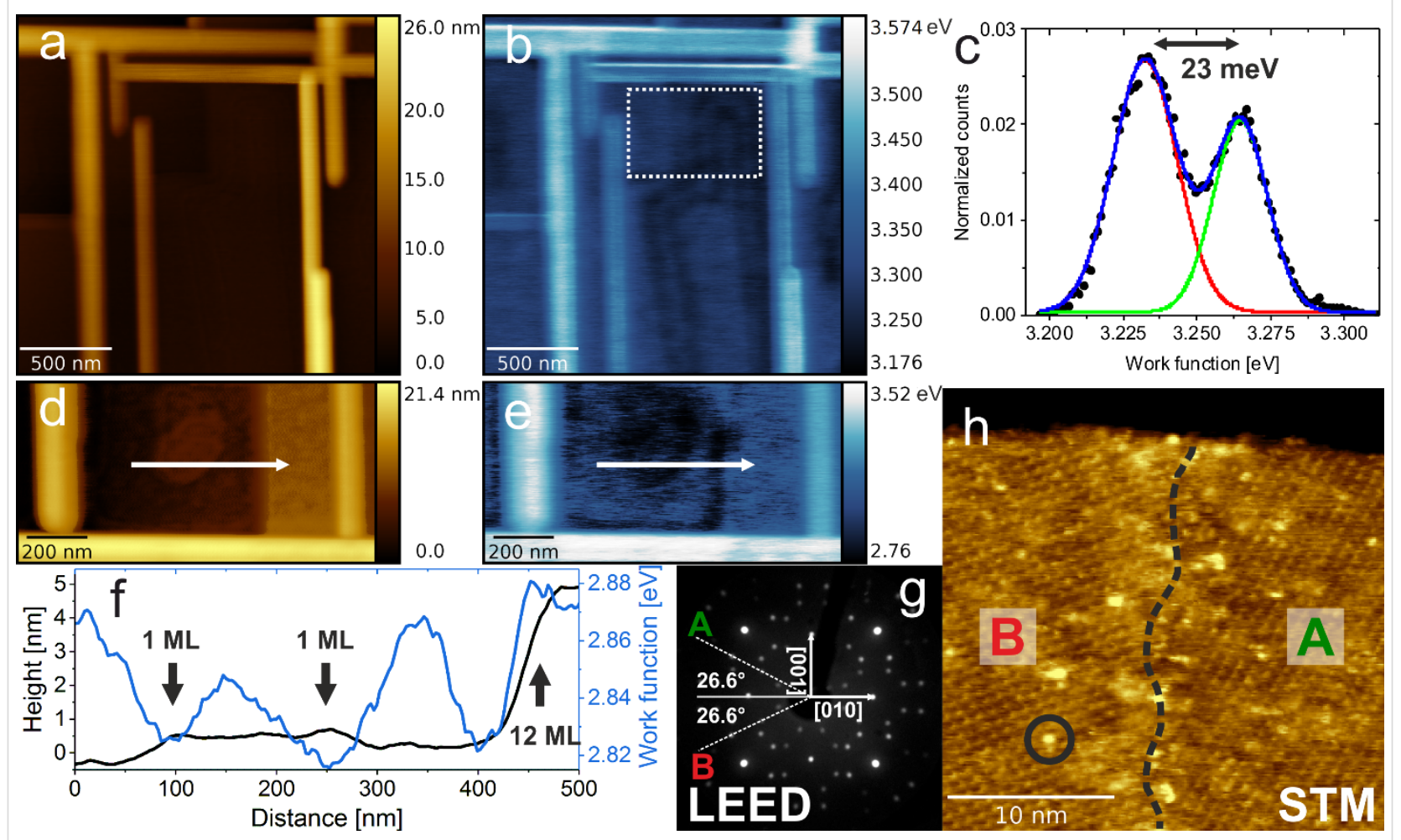

Figure 5: Characterization of the $\mathrm{SrTiO}_{3}(100)$ surface by KPFM. a) Topography of a TiO/SrTiO 3 structure $(\Delta f=-20 \mathrm{~Hz})$, b) corresponding work function map from KPFM, c) work function histogram from the selected area in b), d) and e) topography and work function of a different TiO/STO network $(\Delta f=-20 \mathrm{~Hz})$, showing the WF correlation with step edges. f) Topography and work function line profiles, extracted from d) and e). g) LEED pattern (100 eV beam energy) indicating the perfect $(\sqrt{5} \times \sqrt{5})$ R26.6 $6^{\circ}$ reconstruction with two equiprobable domains $A$ and $B$. $h$ ) STM topography showing the concurrent presence of $A$ and $B$ domains, with defects marked in black.

the next experiment was aimed to study the work function dependence upon controlled reoxidation of reduced oxides.

Transition metal oxide nanostructures find manifold applications, especially in various (photo)catalytic processes, e.g., water splitting $[1,44]$. For industrial uses, the samples have to be exposed to ambient conditions, therefore it is necessary to investigate the impact of air exposure on electronic surface properties. Moreover, transition metal oxides are often regarded as promising materials for sensing applications, due to the strong response to gaseous pressures, especially oxygen and water [45]. The tool of choice that provides information on the subtle changes in the work function is KPFM, which has been employed for research on the photocatalytic activity of $\mathrm{TiO}_{2}$-based doped and undoped nanofibers $[46,47]$ and for the understanding of the operation of nanostructure electrochemical sensors [48].

Thus, we have focused on the systematic approach of the influence of ambient air on the work function of $\mathrm{TiO}$ and $\mathrm{SrTiO}_{3}(100)$. Figure $6 \mathrm{a}$ shows the topography and work function of representative TiO nanowire networks before and after ambient air exposure. Additionally, the impact of low tempera- ture annealing $\left(230^{\circ} \mathrm{C}\right)$ in UHV is presented. After the venting of the vacuum chamber, the $\mathrm{TiO}$ nanowires still had a much higher work function than the $\mathrm{SrTiO}_{3}(100)$ surface; however, the absolute values for both materials increased. It is widely known that water molecules present in the ambient air form a dipole layer, which can give rise to an effective surface potential [49].

To reduce the impact of weakly interacting adsorbates, in the next step, the sample underwent in situ UHV annealing, which implicated a WF decrease of both $\mathrm{TiO}$ and $\mathrm{SrTiO}_{3}$ by about $0.2-0.4 \mathrm{eV}$. To illustrate the experimental findings better, three histograms of reduced, air-oxidized and outgassed work functions are plotted in Figure 6b. It is immediately visible that the general three-peak nature of STO and lower and higher TiO values of the WF are preserved, however the offset changes, as illustrated in Figure 6c. The STO(100) work function increases by $0.9 \mathrm{eV}$ due to air exposure, similar to the case of oxygen reoxidation [30]. A conclusion can be drawn that the main contribution of the work function increase is related to oxygen, which refills vacancies of the reduced $(\sqrt{5} \times \sqrt{5}) \mathrm{R} 26.6^{\circ}$ surface and locally oxidizes titanium cations to regain the $4+$ valence state. 

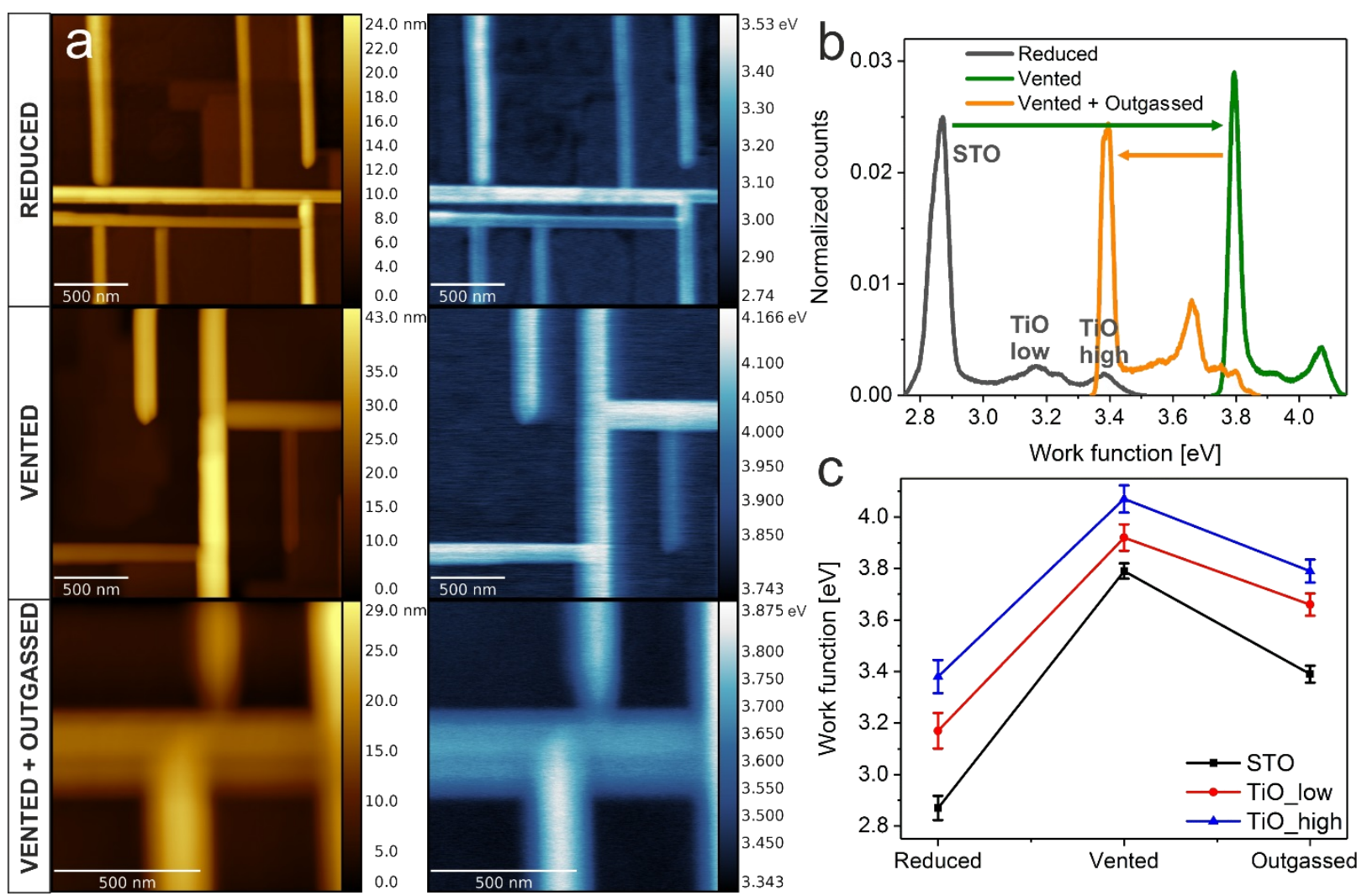

Figure 6: Influence of air exposure on the $\mathrm{TiO} / \mathrm{SrTiO}_{3}$ work function. a) $\mathrm{KPFM}$ topography and work function of a $\mathrm{TiO}^{\mathrm{S}} \mathrm{SrTiO}$, heterostructure $\left(\Delta f=-40 \mathrm{~Hz}\right.$ ) after reduction (first row), exposed to ambient air (second row) and after annealing up to $230{ }^{\circ} \mathrm{C}$ (third row). b) Work function histograms of the presented cases. c) Evolution of the work function value for the $\mathrm{SrTiO}_{3}(100)$ surface (black), lower TiO facets (red) and higher (blue).

Vented TiO nanowires have a work function that is higher by $0.65 \mathrm{eV}$, independent of the face, which is almost $0.5 \mathrm{eV}$ higher than in the case of pure oxygen (not shown). This is a footprint of the high catalytic activity of the TiO surface, which was previously postulated in the case of $\mathrm{TiO} / \mathrm{TiO}_{2}$ nanoparticles [44]. Reduced titania $\mathrm{TiO}_{1.23}$ proves also to be a promising candidate for electrochemical water splitting [50]. As the catalytic activity of a crystalline rock-salt TiO phase has yet to be studied, this is a first indirect observation of the high potential of such a structure.

After in situ annealing to $230{ }^{\circ} \mathrm{C}$, the work function of $\mathrm{TiO}$ nanowires drops by $0.2 \mathrm{eV}$, in contrast to the $0.35 \mathrm{eV}$ decrease for the $\mathrm{SrTiO}_{3}(100)$ surface. A temperature of $230{ }^{\circ} \mathrm{C}$ was chosen to enable desorption of the most abundant adsorbates, also mimicking the typical operating temperatures of metal oxide gas sensors [51]. Numerical calculations suggest that $\mathrm{H}_{2} \mathrm{O}$ and $\mathrm{CO}_{2}$ species present in air saturate almost all free adsorption sites on $\mathrm{TiO}_{2}$-terminated $\mathrm{SrTiO}_{3}(100)$ [52]. The low Redhead desorption temperature of $\mathrm{H}_{2} \mathrm{O}\left(\mathrm{CO}_{2}\right)$ of 233-283 K (428-523 K) calculated by Baniecki et al. [52] suggests that our surface, which is predominantly $\mathrm{TiO}_{2}$-terminated, is cleaned of those adsorbates after annealing, hence the $0.35 \mathrm{eV}$ difference in the WF.

The lower response of $\mathrm{TiO}$ to annealing is a hint of the higher adsorption energy of the interacting gases. It suggests the chemisorption of $\mathrm{H}_{2} \mathrm{O}$ and $\mathrm{CO}_{2}$ and dissociation of their products, stemming from the high catalytic activity of the TiO phase.

To wrap up our experimental findings of the KPFM study of $\mathrm{TiO}$ and $\mathrm{SrTiO}_{3}(100)$ crystals, a comparison between the obtained work functions as well as the subject literature values is presented in Table 1. The first direct work function measurement of the cubic $\gamma$-phase of TiO yields $3.31(21) \mathrm{eV}$ for the as-grown (reduced in $\mathrm{UHV}$ at $1150{ }^{\circ} \mathrm{C}$ ) and $3.75(11) \mathrm{eV}$ for vented and outgassed material, which is most likely close to the value for the pristine $\mathrm{TiO}$ crystal. To date, the only values for the TiO WF come from polycrystalline samples (films, nanoparticles) and from the laterally averaging method of UPS, thus this cannot be reasonably compared with our findings. The WF 


\begin{tabular}{|c|c|c|c|c|}
\hline Material & Sample & WF $[e V]$ & Method & Ref. \\
\hline \multirow[t]{6}{*}{$\mathrm{TiO}$} & TiO nanoparticles (14 nm) & 3.01 & UPS & [44] \\
\hline & TiO thin polycrystalline film & $\approx 4.5$ & UPS & [53] \\
\hline & TiO cubic $Y$-phase (nanowires): & & & \\
\hline & $\cdot$ reduced $\left(\mathrm{UHV}, 1150^{\circ} \mathrm{C}\right)$ & $3.31(21)$ & KPFM & this study \\
\hline & - air-exposed & $3.92(17)$ & & \\
\hline & - air-exposed and annealed & $3.75(11)$ & & \\
\hline \multirow[t]{12}{*}{$\mathrm{SrTiO}_{3}$} & $\mathrm{SrTiO}_{3}(100)$ & 4.13 & XPEEM & [32] \\
\hline & $\mathrm{SrTiO}_{3}(110)$ & 4.32 & & \\
\hline & $\mathrm{SrTiO}_{3}(111)$ & 4.34 & & \\
\hline & $\mathrm{SrTiO}_{3}(100)$ & 4.2 & UPS & [33] \\
\hline & $\mathrm{SrTiO}_{3}(100)$ reduced at $900{ }^{\circ} \mathrm{C}$ & $3.478(64)$ & KPFM & [30] \\
\hline & $\begin{array}{l}\mathrm{TiO}_{2} \text {-terminated } \mathrm{SrTiO}_{3}(100) \\
\text { SrO-terminated } \mathrm{SrTiO}_{3}(100)\end{array}$ & $\begin{array}{l}4.2-4.3 \\
2.5-3.0\end{array}$ & macroscopic Kelvin probe & [54] \\
\hline & $\mathrm{SrTiO}_{3}(100)$ (single crystal): & & & \\
\hline & $\cdot$ reduced $\left(\mathrm{UHV}, 1150^{\circ} \mathrm{C}\right)$ & $3.12(18)$ & KPFM & this study \\
\hline & - air-exposed & $3.835(72)$ & & \\
\hline & $\cdot$ air-exposed and annealed & & & \\
\hline & $\begin{array}{l}\mathrm{SrTiO}_{3}(100) \mathrm{BHF} \text {-etched, air-annealed }\left(100{ }^{\circ} \mathrm{C}\right) \\
\cdot \phi_{\mathrm{TiO}}-\Phi_{\text {Sro difference }}\end{array}$ & $\approx 10 \mathrm{meV}$ & KPFM & [41] \\
\hline & $\begin{array}{l}\mathrm{SrTiO}_{3}(100) \mathrm{BHF} \text { etched, air annealed }\left(1300^{\circ} \mathrm{C}\right) \\
\cdot \Phi_{\mathrm{TiO} 2}-\phi_{\text {Sro }} \text { difference }\end{array}$ & $\approx 0.5 \mathrm{eV}$ & LEEM & [55] \\
\hline
\end{tabular}

value obtained for the reduced $\mathrm{SrTiO}_{3}(100)$ surface of 3.12(18) $\mathrm{eV}$ follows the trend of a decreasing work function upon reduction and an increasing one due to reoxidation [30]. It is worth mentioning that substantial discrepancies between the measured WF for transition metal oxides, besides the different quality of the samples, could stem from the suboptimal conditions during preparation, e.g., holder materials can act as oxygen getters, influencing the reduction state of a sample [12].

\section{Conclusion}

We have presented a thorough study of the Kelvin probe force microscopy investigations on the electronic properties of cubic $\mathrm{TiO}$ formed on a $\mathrm{SrTiO}_{3}(100)$ single crystal surface. To our best knowledge, this is the first measurement of the crystalline $\mathrm{TiO}$ work function and its dependence on the gaseous pressure of air in comparison to the $\mathrm{SrTiO}_{3}(100)$ response. The rock-salt cubic $\gamma$-phase, with a high crystallographic order, has a work function of 3.31(21) eV with significant variations on the surface, which are related to the different facets exposed. The higher work function of TiO compared to $\mathrm{STO}(100)$ is accompanied with a higher conductivity, with an ohmic behavior, which was checked by simultaneous LC-AFM measurements. Full ambient venting results in a $0.6 \mathrm{eV}$ increase in the $\mathrm{TiO}$ work function and is a hint of the ongoing catalyzed dissociation of $\mathrm{CO}_{2}$ and $\mathrm{H}_{2} \mathrm{O}$ present in ambient air. The work function of TiO decreases by $0.2 \mathrm{eV}$ as a result of $230{ }^{\circ} \mathrm{C}$ UHV annealing, being a measure of the amount of weakly adsorbed species. Air exposure of the $(\sqrt{5} \times \sqrt{5}) \mathrm{R} 26.6^{\circ} \mathrm{TiO}_{2}$-terminated $\mathrm{SrTiO}_{3}(100)$ sur- face results in a $0.9 \mathrm{eV}$ increase in the work function, which is similar to the increase observed for reoxidation in pure oxygen. Annealing induces a decrease of $0.4 \mathrm{eV}$ as a result of the desorption of weakly bonded species.

Our study also contains an estimation of the FM-KPFM lateral resolution, which enabled reliable measurements of TiO nanowires separated by $40 \mathrm{~nm}$, proving the suitability of the KPFM technique for the investigation of transition metal oxide structures, even with remarkable topographical variations.

In conclusion, the presented results acquired for crystalline $\mathrm{TiO}$ and $\mathrm{SrTiO}_{3}$ phases provide important information on the work function values themselves but also on their interaction with air and operation upon elevated temperatures.

\section{Experimental}

We investigated Verneuil-grown epitaxy-ready-polished $\mathrm{SrTiO}_{3}(100)$ crystals, provided by the Crystec company. The crystals were mounted onto Omicron direct heating holders and introduced into the UHV chamber, with a base pressure of $5 \times 10^{-10}$ mbar, maintained by the use of turbo-molecular, ion and sublimation pumps. In order to produce a $\mathrm{TiO}$ nanowire network on the surface of STO(100), the extremely low oxygen partial pressure (ELOP) method of perovskite decomposition was adapted (for more information see $[12,26])$. The $\mathrm{SrTiO}_{3}(100)$ single crystal $\left(10 \times 3 \times 0.5 \mathrm{~mm}^{3}\right)$ sample was mounted on an n-doped $\mathrm{Si}(111)$ single crystal, which acted as 
an oxygen getter, allowing extremely low effective oxygen partial pressures to be reached on the STO surface (below $10^{-20}$ mbar). As a result of the high-temperature reduction $\left(1150{ }^{\circ} \mathrm{C}, 1 \mathrm{~h}\right)$ under such conditions, a macroscopic network of nanowires was formed. After slow cooling down to room temperature, the samples were transferred in situ to the microscope chamber of an Omicron RT STM/AFM system, where KPFM, LC-AFM and STM measurements were performed. KPFM, operating in FM mode, was used with a single-pass method, with three feedback loops maintaining the oscillation amplitude, phase and frequency shift [56]. The real oscillation amplitude was in the range of $10 \mathrm{~nm}$. In order to obtain the CP D signal, an AC-modulated bias was applied to the bottom of the electrode, where a sample was mounted. The modulation frequency and amplitude were set to $315 \mathrm{~Hz}$ and $500 \mathrm{mV}$, respectively.

In order to ensure reproducible FM-KPFM results, two different types of AFM cantilevers were used: PPP-ContPt (PtIrcoated) and PtSi-FM (platinum silicide tips), both from Nanosensors. Such cantilevers are widely used as conducting tips in a contact mode AFM, allowing for a high lateral resolution in conductivity measurements. The remarkable mechanical stability of the selected cantilevers allowed for the noncontact mode measurements (with a Kelvin loop) using the very same tip, maintaining oscillations at the higher harmonics of the fundamental frequency $(\approx 75 \mathrm{kHz})$. Hence, in order to record current and CPD maps from the very same sample area, KPFM measurements were first performed with the soft cantilever forced to oscillate at higher harmonics, then the tip was retracted tens of nanometers from the surface, all feedback loops were turned down and a contact mode AFM scan was performed when approached with a single loop maintaining a deflection set point of 10-30 mV. The high conductivity of both $\mathrm{TiO}$ and STO materials enabled a low sample bias of $+1 \mathrm{mV}$ for the LC-AFM measurements to be used.

Real work function values were calculated from recorded CPD maps after calibration against a material of known work function, here highly oriented pyrolytic graphite (HOPG), which was annealed in situ up to $300{ }^{\circ} \mathrm{C}$ in order to remove water and other adsorbates. After each set of KPFM measurements, the HOPG surface was scanned employing the same experimental parameters and a $V_{\mathrm{CPD}}(\mathrm{HOPG})$ was obtained. Assuming a $\mathrm{HOPG}$ work function of $\phi_{\mathrm{HOPG}}=4.5 \mathrm{eV}$ (subject literature values: $4.6 \pm 0.1 \mathrm{eV}$ [57], $4.4 \pm 0.1 \mathrm{eV}$ [58]), a work function of $\mathrm{TiO} / \mathrm{STO}$ was evaluated by applying the equation: $\phi_{\mathrm{TiO} / \mathrm{STO}}=V_{\mathrm{CPD}}(\mathrm{TiO} / \mathrm{STO})-V_{\mathrm{CPD}}(\mathrm{HOPG})+\phi_{\mathrm{HOPG}}$

Ambient air exposure was realized by venting the sample in the load-lock and then introducing it again into the UHV system.
Subsequent annealing up to $230^{\circ} \mathrm{C}$ for $1 \mathrm{~h}$ hour enabled the estimation of the content of the weakly adsorbed species at the surface.

\section{Acknowledgements}

Support by the Polish National Science Center (UMO- 2018/29/ $\mathrm{B} / \mathrm{ST}$ /01406) is acknowledged.

\section{ORCID ${ }^{\circledR}$ iDs}

Dominik Wrana - https://orcid.org/0000-0002-8239-0043 Karol Cieślik - https://orcid.org/0000-0002-1069-1840 Wojciech Belza - https://orcid.org/0000-0003-1959-3160 Christian Rodenbücher - https://orcid.org/0000-0001-8029-3066 Krzysztof Szot - https://orcid.org/0000-0001-8773-2754 Franciszek Krok - https://orcid.org/0000-0002-6931-3545

\section{Preprint}

A non-peer-reviewed version of this article has been previously published as a preprint doi:10.3762/bxiv.2019.12.v1

\section{References}

1. Imran, M.; Yousaf, A. B.; Zhou, X.; Jiang, Y.-F.; Yuan, C.-Z.; Zeb, A.; Jiang, N.; Xu, A.-W. J. Phys. Chem. C 2017, 121, 1162-1170. doi:10.1021/acs.jpcc.6b10274

2. Compton, R., Ed. Electrode kinetics: reactions; Elsevier: Amsterdam, Netherlands, 1987; Vol. 27.

3. Waser, R.; Dittmann, R.; Staikov, G.; Szot, K. Adv. Mater. (Weinheim, Ger.) 2009, 21, 2632-2663. doi:10.1002/adma.200900375

4. Yu, X.; Marks, T. J.; Facchetti, A. Nat. Mater. 2016, 15, 383-396. doi:10.1038/nmat4599

5. Wrana, D.; Kratzer, M.; Szajna, K.; Nikiel, M.; Jany, B. R.; Korzekwa, M.; Teichert, C.; Krok, F. J. Phys. Chem. C 2015, 119, 17004-17015. doi:10.1021/acs.jpcc.5b04384

6. Lin, Y.; Norman, C.; Srivastava, D.; Azough, F.; Wang, L.; Robbins, M.; Simpson, K.; Freer, R.; Kinloch, I. A. ACS Appl. Mater. Interfaces 2015, 7, 15898-15908. doi:10.1021/acsami.5b03522

7. Jöhr, R.; Hinaut, A.; Pawlak, R.; Sadeghi, A.; Saha, S.; Goedecker, S.; Such, B.; Szymonski, M.; Meyer, E.; Glatzel, T. J. Chem. Phys. 2015, 143, 094202. doi:10.1063/1.4929608

8. Fatayer, S.; Schuler, B.; Steurer, W.; Scivetti, I.; Repp, J.; Gross, L.; Persson, M.; Meyer, G. Nat. Nanotechnol. 2018, 13, 376-380. doi:10.1038/s41565-018-0087-1

9. Axt, A.; Hermes, I. M.; Bergmann, V. W.; Tausendpfund, N.; Weber, S. A. L. Beilstein J. Nanotechnol. 2018, 9, 1809-1819. doi:10.3762/bjnano.9.172

10. Batko, I.; Batkova, M. Eur. Phys. J.: Appl. Phys. 2012, 58, 20102. doi:10.1051/epjap/2012120102

11. Zerweck, U.; Loppacher, C.; Otto, T.; Grafström, S.; Eng, L. M. Phys. Rev. B 2005, 71, 125424. doi:10.1103/physrevb.71.125424

12. Wrana, D.; Rodenbücher, C.; Jany, B. R.; Kryshtal, O.; Cempura, G.; Kruk, A.; Indyka, P.; Szot, K.; Krok, F. Nanoscale 2019, 11, 89-97. doi:10.1039/c8nr04545c

13. Su, J.; Zou, X.; Chen, J.-S. RSC Adv. 2014, 4, 13979-13988. doi:10.1039/c3ra47757f 
14. Zhong, Z.; Hansmann, P. Phys. Rev. B 2016, 93, 235116. doi:10.1103/physrevb.93.235116

15. Zhang, T.-F.; Tang, X.-G.; Huang, X.-X.; Liu, Q.-X.; Yiang, Y.-P.; Zhou, Q.-F. Energy Technol. 2016, 4, 633-640. doi:10.1002/ente.201500436

16. Ohtomo, A.; Hwang, H. Y. Nature 2004, 427, 423-426. doi:10.1038/nature02308

17. Song, Q.; Zhang, H.; Su, T.; Yuan, W.; Chen, Y.; Xing, W.; Shi, J.; Sun, J.; Han, W. Sci. Adv. 2017, 3, e1602312. doi:10.1126/sciadv.1602312

18. Yamashita, J. J. Phys. Soc. Jpn. 1963, 18, 1010-1016. doi:10.1143/jpsj.18.1010

19. Shu, G.; Wang, H.; Zhao, H.-X.; Zhang, X. ACS Appl. Mater. Interfaces 2019, 11, 3323-3333. doi:10.1021/acsami.8b17418

20. Xu, J.; Wang, D.; Yao, H.; Bu, K.; Pan, J.; He, J.; Xu, F.; Hong, Z.; Chen, X.; Huang, F. Adv. Mater. (Weinheim, Ger.) 2018, 30, 1706240. doi:10.1002/adma.201706240

21.Zhang, C.; Hao, F.; Gao, G.; Liu, X.; Ma, C.; Lin, Y.; Yin, Y.; Li, X. npj Quantum Mater. 2017, 2, 2. doi:10.1038/s41535-016-0006-3

22. Wang, D.; Huang, C.; He, J.; Che, X.; Zhang, H.; Huang, F. ACS Omega 2017, 2, 1036-1039. doi:10.1021/acsomega.7b00048

23. Shirotori, Y.; Sawada, K.; Ozawa, K.; Edamoto, K. Thin Solid Films 2004, 464-465, 76-79. doi:10.1016/j.tsf.2004.06.086

24. Pabón, B. M.; Beltrán, J. I.; Sánchez-Santolino, G.; Palacio, I.; López-Sánchez, J.; Rubio-Zuazo, J.; Rojo, J. M.; Ferrer, P.; Mascaraque, A.; Muñoz, M. C.; Varela, M.; Castro, G. R.; de la Fuente, O. R. Nat. Commun. 2015, 6, 6147. doi:10.1038/ncomms7147

25. Amano, S.; Bogdanovski, D.; Yamane, H.; Terauchi, M.; Dronskowski, R. Angew. Chem., Int. Ed. 2016, 55, 1652-1657. doi:10.1002/anie.201510479

26. Rodenbücher, C.; Meuffels, P.; Speier, W.; Ermrich, M.; Wrana, D.; Krok, F.; Szot, K. Phys. Status Solidi RRL 2017, 11, 1700222. doi:10.1002/pssr.201700222

27. Banus, M. D.; Reed, T. B.; Strauss, A. J. Phys. Rev. B 1972, 5 , 2775-2784. doi:10.1103/physrevb.5.2775

28. Szot, K.; Bihlmayer, G.; Speier, W. Solid State Phys. 2014, 65, 353-559. doi:10.1016/b978-0-12-800175-2.00004-2

29. Rodenbücher, C.; Wrana, D.; Meuffels, P.; Rogala, M.; Krok, F.; Szot, K. APL Mater. 2018, 6, 066105. doi:10.1063/1.5028424

30. Wrana, D.; Rodenbücher, C.; Bełza, W.; Szot, K.; Krok, F. Appl. Surf. Sci. 2018, 432, 46-52. doi:10.1016/j.apsusc.2017.06.272

31. Zhong, Z.; Kelly, P. J. EPL 2008, 84, 27001. doi:10.1209/0295-5075/84/27001

32. Zagonel, L. F.; Bäurer, M.; Bailly, A.; Renault, O.; Hoffmann, M.; Shih, S.-J.; Cockayne, D.; Barrett, N. J. Phys.: Condens. Matter 2009, 21, 314013. doi:10.1088/0953-8984/21/31/314013

33. Chung, Y.-W.; Weissbard, W. B. Phys. Rev. B 1979, 20, 3456-3461. doi:10.1103/physrevb.20.3456

34. Sadewasser, S.; Glatzel, T.; Rusu, M.; Jäger-Waldau, A.; Lux-Steiner, M. C. Appl. Phys. Lett. 2002, 80, 2979-2981. doi:10.1063/1.1471375

35. Imanishi, A.; Tsuji, E.; Nakato, Y. J. Phys. Chem. C 2007, 111, 2128-2132. doi:10.1021/jp0668403

36. Polak, L.; Wijngaarden, R. J. Ultramicroscopy 2016, 171, 158-165. doi:10.1016/j.ultramic.2016.09.014

37. Krok, F.; Sajewicz, K.; Konior, J.; Goryl, M.; Piatkowski, P.; Szymonski, M. Phys. Rev. B 2008, 77, 235427. doi:10.1103/physrevb.77.235427
38. Sadewasser, S.; Leendertz, C.; Streicher, F.; Lux-Steiner, M. C. Nanotechnology 2009, 20, 505503. doi:10.1088/0957-4484/20/50/505503

39. Jang, Y. H.; Cho, J. H. J. Korean Phys. Soc. 2016, 69, 783-788. doi:10.3938/jkps.69.783

40. Martirez, J. M. P.; Morales, E. H.; Saidi, W. A.; Bonnell, D. A.; Rappe, A. M. Phys. Rev. Lett. 2012, 109, 256802. doi:10.1103/physrevlett.109.256802

41. Gellé, F.; Chirita, R.; Mertz, D.; Rastei, M. V.; Dinia, A.; Colis, S. Surf. Sci. 2018, 677, 39-45. doi:10.1016/j.susc.2018.06.001

42. Sasahara, A.; Uetsuka, H.; Onishi, H. Jpn. J. Appl. Phys., Part 1 2004, 43, 4647-4650. doi:10.1143/jjap.43.4647

43. Martinez, U.; Hansen, J. Ø.; Lira, E.; Kristoffersen, H. H.; Huo, P.; Bechstein, R.; Lægsgaard, E.; Besenbacher, F.; Hammer, B.; Wendt, S. Phys. Rev. Lett. 2012, 109, 155501. doi:10.1103/physrevlett.109.155501

44. Chen, Z.; Zhong, W.; Liang, Z.; Li, W.; He, G.; Wang, Y.; Li, W.; Xie, Y.; He, Q. J. Nanomater. 2014, 2014, 298619. doi:10.1155/2014/298619

45. Henning, A.; Günzburger, G.; Jöhr, R.; Rosenwaks, Y.; Bozic-Weber, B.; Housecroft, C. E.; Constable, E. C.; Meyer, E.; Glatzel, T. Beilstein J. Nanotechnol. 2013, 4, 418-428. doi:10.3762/bjnano.4.49

46. Wu, M.-C.; Liao, H.-C.; Cho, Y.-C.; Tóth, G.; Chen, Y.-F.; Su, W.-F.; Kordás, K. J. Mater. Chem. A 2013, 1, 5715-5720. doi:10.1039/c3ta01011b

47. Wu, M.-C.; Liao, H.-C.; Cho, Y.-C.; Hsu, C.-P.; Lin, T.-H.; Su, W.-F.; Sápi, A.; Kukovecz, Á.; Kónya, Z.; Shchukarev, A.; Sarkar, A.; Larsson, W.; Mikkola, J.-P.; Mohl, M.; Tóth, G.; Jantunen, H.; Valtanen, A.; Huuhtanen, M.; Keiski, R. L.; Kordás, K. J. Nanopart. Res. 2014, 16, 2143. doi:10.1007/s11051-013-2143-y

48. Henning, A.; Rosenwaks, Y. KPFM of Nanostructured Electrochemical Sensors. In Kelvin Probe Force Microscopy; Sadewasser, S.; Glatzel, T., Eds.; Springer: Cham, Switzerland, 2018; pp 367-389. doi:10.1007/978-3-319-75687-5_12

49. Kim, H.; Kim, D.-W. Appl. Phys. A: Mater. Sci. Process. 2011, 102 , 949-953. doi:10.1007/s00339-011-6277-4

50. Swaminathan, J.; Subbiah, R.; Singaram, V. ACS Catal. 2016, 6, 2222-2229. doi:10.1021/acscatal.5b02614

51. Wang, C.; Yin, L.; Zhang, L.; Xiang, D.; Gao, R. Sensors 2010, 10, 2088-2106. doi:10.3390/s100302088

52. Baniecki, J. D.; Ishii, M.; Kurihara, K.; Yamanaka, K.; Yano, T.; Shinozaki, K.; Imada, T.; Kobayashi, Y. J. Appl. Phys. 2009, 106, 054109. doi:10.1063/1.3169654

53. Greiner, M. T.; Chai, L.; Helander, M. G.; Tang, W.-M.; Lu, Z.-H. Adv. Funct. Mater. 2012, 22, 4557-4568. doi:10.1002/adfm.201200615

54. Susaki, T.; Makishima, A.; Hosono, H. Phys. Rev. B 2011, 84, 115456. doi:10.1103/physrevb.84.115456

55. Jobst, J.; Boers, L. M.; Yin, C.; Aarts, J.; Tromp, R. M.; van der Molen, S. J. Ultramicroscopy 2019, 200, 43-49. doi:10.1016/j.ultramic.2019.02.018

56. Krok, F.; Kolodziej, J. J.; Such, B.; Czuba, P.; Struski, P.; Piatkowski, P.; Szymonski, M. Surf. Sci. 2004, 566-568, 63-67. doi:10.1016/j.susc.2004.05.023

57. Melitz, W.; Shen, J.; Lee, S.; Lee, J. S.; Kummel, A. C.; Droopad, R.; Yu, E. T. J. Appl. Phys. 2010, 108, 023711. doi:10.1063/1.3462440

58. Jeong, H. K.; Yang, C.; Kim, B. S.; Kim, K.-j. EPL 2010, 92, 37005. doi:10.1209/0295-5075/92/37005 


\section{License and Terms}

This is an Open Access article under the terms of the Creative Commons Attribution License (http://creativecommons.org/licenses/by/4.0). Please note that the reuse, redistribution and reproduction in particular requires that the authors and source are credited.

The license is subject to the Beilstein Journal of Nanotechnology terms and conditions: (https://www.beilstein-journals.org/bjnano)

The definitive version of this article is the electronic one which can be found at:

doi:10.3762/bjnano.10.155 